\title{
Creencias de formadores de profesores de matemática sobre resolución de problema
}

\section{Mathematics Teacher Educator's Beliefs on Problem Solving}

\author{
Eugenio Chandia* \\ Daniela Rojas ${ }^{* *}$ \\ Francisco Rojas ${ }^{* * *}$ \\ Sebastián Howard ${ }^{* * * *}$
}

\begin{abstract}
Resumen
La resolución de problemas es considerada como una de las principales oportunidades de aprendizaje matemático, especialmente en la formación inicial de profesores, donde los formadores juegan un rol fundamental sobre su comprensión. Considerando al menos tres enfoques en la resolución de problemas (proceso, método y objetivo instruccional), esta investigación da cuenta de las creencias de un grupo de formadores de profesores de educación básica (6-12 años) respecto de esta característica de la actividad matemática. Para ello, se utilizó una metodología de viñetas, en la cual se cuestiona al formador cómo trabajar con sus estudiantes la resolución de problemas. Los formadores declaran que la resolución de problemas implica un proceso que está compuesto por etapas o fases, aunque el análisis, la ejecución y la misma comprobación de la estrategia de resolución e interacción de estas fases no se observan en sus declaraciones.
\end{abstract}

Palabras-clave: Resolución de Problemas. Formador de Profesores de Matemática. Creencias.

\begin{abstract}
Problem solving is seen as a major mathematical learning opportunity, especially in the pre-service teacher education, where educators play a key role in the students understanding. Considering at least three approaches to problem solving (process, objective method of instruction), this research addresses the beliefs of a group of teacher educators for elementary levels (ages 6-12) for this feature of mathematical activity. In order to do so, a methodology of vignettes was used, in which educators were asked to talk about their work on problem solving with students. Teacher educators say problem solving involves a process that comprises the steps or phases, although the analysis, implementation and verification of resolution strategies and the interaction of these phases was not observed in their statements.
\end{abstract}

\footnotetext{
* Estudiante Doctoral del programa Doctorado en Ciencias de la Educación en la Facultad de Educación de la Pontificia Universidad Católica de Chile (PUC), Santigo, Región Metropolitana, Chile. Dirección postal: Av. Vicuña Mackenna 4860, Macul, 7820436, Santiago, Chile. E-mail: echandia@uc.cl

** Estudiante Doctoral del programa Doctorado en Ciencias de la Educación en la Facultad de Educación de la Pontificia Universidad Católica de Chile. (PUC), Santiago, Región Metropolitana, Chile. Dirección postal: Av. Vicuña Mackenna 4860, Macul, 7820436, Santiago, Chile. E-mail: dhrojas1@uc.cl

**** Doctor en Didáctica de la Matemática y las Ciencias Experimentales por la Universitat Autònoma de Barcelona (UAB). Profesor Asistente en la Facultad de Educación de la Pontificia Universidad Católica de Chile, Santiago, Chile. Dirección postal: Av. Vicuña Mackenna 4860, Macul, 7820436, Santiago, Chile. E-mail: frojass@uc.cl

Doctor en Ciencias de la Educación por la Pontificia Universidad Católica de Chile (PUC). Profesor Asistente en la Facultad de Educación de la Universidad Diego Portales, Santiago, Región Metropolitana, Chile. Dirección postal: Vergara 210, Barrio Universitario, 8370067, Santiago, Chile. E-mail: sebastian.howard@udp.cl
} 
Keywords: Problem Solving. Mathematics Teacher Educators. Beliefs.

\section{Introducción}

Dentro de las características clave de la actividad matemática escolar, la resolución de problemas es considerada como una de las principales formas de acceder a un conocimiento matemático de calidad. Esto impone un enorme desafío no solo a la escuela y sus profesores, sino también a los contextos de formación inicial. Construir un conocimiento profesional sobre resolución de problemas requiere un fuerte componente disciplinar de parte de los futuros profesores, pero también competencias didácticas del formador de docentes para el diseño y trabajo de tareas que permitan que esto ocurra. Sin embargo, las creencias que tienen estos actores sobre matemática, su enseñanza y aprendizaje, mediadas fundamentalmente por su experiencia escolar y universitaria, afectarán qué entienden por resolución de problemas y las formas en que se desarrolla ese conocimiento profesional. En particular, en este artículo queremos explorar las creencias de los formadores de profesores sobre este componente de la actividad matemática, indagando en las justificaciones que dan a las formas en que lo trabajan en el aula universitaria, específicamente en contexto de formación inicial de profesores de educación básica (6-12 años).

\section{Antecedentes}

En la formación de profesores de matemática, se espera que exista una orquestación de los diferentes tipos de conocimiento profesional que requiere un profesor para enseñar matemática (BALL; THAMES; PHELPS, 2008). Para construir dicho conocimiento, en la formación inicial deberían coexistir ciertas oportunidades de aprendizaje, subyacentes a cualquier proceso formativo (ROJAS; DEULOFEU, 2015). Por una parte, el cuerpo de formadores debería ofrecer a los futuros profesores oportunidades de aprender matemáticas tal como se espera que sus alumnos las aprendan (CHAPMAN, 2008; DEULOFEU; FIGUEIRAS; PUJOL, 2011; GÓMEZ-CHACÓN, 2005), generando, así, procesos de modelación de la práctica de enseñanza. Por otra, el formador debería plantear actividades que fueran oportunidades de aprender a enseñar matemáticas, en el sentido de planificar la enseñanza, analizar la gestión a través de episodios de aula y trabajar a partir de realizaciones 
de alumnos, estableciendo una fuerte relación teoría-práctica (BOYD et al., 2009; GELLERT, 2005).

Si bien la información entregada por TEDS-M (TATTO et al., 2012) ha permitido incorporar información relevante en los procesos formativos al interior de las universidades e instituciones formadoras de profesores, aún no tenemos mucho conocimiento de lo que ocurre dentro de dichos contextos, ni como esta información ha sido utilizada. En el contexto nacional, el Informe de la Comisión sobre Formación Inicial Docente (MINEDUC, 2005) deja en evidencia la desvinculación entre la formación de profesores y la realidad escolar (programas de formación sobrecargados, desarticulación entre formación disciplinar y profesional, escasa relación entre teoría y práctica), lo que se refleja también en la falta de investigaciones de los procesos formativos al interior del aula universitaria (la llamada caja negra) (DOMÍNGUEZ; MECKES, 2011; OCDE; BANCO MUNDIAL, 2009; SOTOMAYOR; GYSLING, 2011), ni de la relación entre formadores y estudiantes (COCHRAN-SMITH; FRIES; ZEICHNER, 2006). Dentro de este escenario, una de las dimensiones poco abordadas en la investigación ha sido el formador de profesores, siendo este un actor clave en los procesos de formación inicial, pues es quien tiene a cargo dicho proceso formativo y su indagación (CISTERNAS, 2011).

En el proceso de formación de profesores, los formadores tienen como objetivo que el futuro profesor construya un marco de conocimiento (saberes y habilidades), adquiera una cierta experiencia concerniente a las distintas etapas del proceso de enseñanza-aprendizaje (CHRISTIANSEN; WALTER, 1986), y permita desarrollar y mejorar la enseñanza de la disciplina (JAWORSKI, 2008). No obstante, las creencias sobre la actividad matemática escolar de los profesores en formación, que impactan fuertemente en la construcción de su marco de conocimiento, surgen principalmente de su experiencia como alumnos (DEULOFEU; MÁRQUEZ; SANTMARTÍ, 2010), lo cual dificulta la introducción de nuevas perspectivas curriculares y didácticas (e.g. resolución de problemas o enseñanza por competencias), principalmente porque los futuros profesores no han aprendido matemáticas bajo esos nuevos modelos didácticos que se espera que apliquen en la enseñanza de las matemáticas (CHAPMAN, 2008; DEULOFEU; FIGUEIRAS; PUJOL, 2011).

Por ello, creemos que las actividades matemático-didácticas que diseña e implementa el formador - resolución de problemas matemáticos, diseño de secuencias de enseñanza, estudio y reflexión de la práctica, lecturas de referentes teóricos, entre otros (WATSON; MASON, 2007) - junto a sus prácticas instruccionales, deberían permitirles a los estudiantes para profesor construir el conocimiento necesario para enseñar matemáticas en la escuela. De 
hecho, una parte importante del desarrollo de estas actividades o tareas profesionales depende de cómo el formador las gestiona, es decir, de las maneras en que el formador usa las tareas para mostrar los enfoques didáctico-matemáticos subyacentes, así como las características personales que considera que los estudiantes deberían desarrollar (ZASLAVSKY, 2007).

Esta modelación que el formador hace para mostrar los enfoques de enseñanza que espera que sus estudiantes construyan, está mediada por el sistema de creencias de estos actores, en especial por el del formador, entendiendo las creencias como las disposición a actuar de una determinada manera y no solo como una verbalización de lo que se cree (WILSON; COONEY, 2002). Este sistema de creencias, compuesto por aquellas relativas a la educación matemática en general, el sentido de autoeficacia y las asociadas al contexto social (OP'T EYNDE; DE CORTE; VERSCHAFFEL, 2002), es usado como metáfora para representar cómo el conocimiento subjetivo, basado en la experiencia y a menudo implícito, está estructurado.

Al respecto de dicha estructura, Zapata, Blanco y Camacho (2012) sostienen que los estudiantes para profesor otorgan gran importancia a la presencia de la matemática en la sociedad, siendo igual de relevantes las distintas modalidades de esta disciplina (pura y aplicada), considerando el desarrollo del pensamiento lógico matemático como su finalidad. Sin embargo, en el estudio realizado por sus autores existen visones contrapuestas respecto de la enseñanza de las matemáticas: usando métodos formales y deductivos o, por el contrario, usando métodos empíricos, heurísticas y resolución de problemas. Estas dos clases de creencias coinciden con las definidas por Vila (2001), quien señala que existe una visión de la enseñanza de las matemáticas que usa los problemas como subsidiarios de los contenidos (reducción de problemas a no-problemas), a cambio de una visión que usa los problemas como herramienta didáctica focalizada en el desarrollo del pensamiento matemático. Esta discrepancia, también vista entre el perfil de lo que se busca como profesor de matemáticas y aquello que los estudiantes para profesor piensan de las matemáticas (CAMACHO; SOCAS; HERNÁNDEZ, 1998), se presenta como un desafío para los formadores en orden a apoyar el cambio de creencias de los estudiantes (ZAPATA; BLANCO; CAMACHO, 2012), considerando la estabilidad de estas debido a la dificultad de su modificación (LILJEDAHL; OESTERLE; BERNÈCHE, 2012).

Por lo anterior, creemos que entre todos los conocimientos y habilidades a desarrollar en la formación inicial, uno que consideramos clave desde la educación matemática es la resolución de problemas, ya que además de ser una forma de enseñanza, se puede considerar 
un tipo de tarea matemática en sí mismo (YEE, 2006) y, por lo tanto, un tipo de tarea profesional.

Diversas investigaciones han mostrado que los futuros profesores carecen de las habilidades y conocimientos necesarios para determinar qué estrategias usar, cuándo y cómo usarlas para resolver problemas matemáticos (PUTNAM; BORKO, 2000; RANDI; CORNO, 2000), lo cual explicaría que lo que se plantea como resolución de problemas en la formación inicial de profesores está lejos de serlo (FELMER; PERDOMO, 2014). Esto se evidencia en la cantidad y calidad de oportunidades de aprendizaje respecto de la resolución de problemas que se ofrecen a los futuros profesores, lo cual los formadores consideran como bajas o nulas.

Por otra parte, y a nivel estructural, es claro que las líneas de cursos disciplinares en los programas de formación docente afectan más que las líneas didáctica o pedagógica al sistema de creencias que se relacionan con la resolución de problemas en los estudiantes de pedagogía (GINÉ; DEULOFEU, 2014), lo que implica una revisión curricular de la formación inicial.

De este modo, lo que persigue este artículo es dar orientaciones para comprender cuáles son las creencias de los formadores de profesores respecto de la resolución de problemas matemáticos escolares, cuáles son las formas que usan para orquestar las oportunidades de aprender tales conocimientos y habilidades, y saber finalmente en qué lo fundamentan.

\section{Marco de referencia}

La resolución de problemas matemáticos (RPM), en las últimas tres décadas, ha presentado un creciente interés en el mundo de la educación matemática. En particular, ha sido incorporado en los currículos de diversos países. Por ejemplo, en la década de 1990 Estados Unidos declara que la resolución de problemas debiera ser el eje central del currículo escolar (NCTM, 1989, 2000) dada la importancia que tiene para generar habilidades y conocimiento matemáticos. Por su parte, en esa misma década, Chile incorpora al currículo nacional la RPM como contenido mínimo obligatorio. Posteriormente, con las Bases Curriculares (MINEDUC, 2012) la RPM se establece como habilidad transversal a todos los contenidos matemáticos, y se incorpora a los estándares orientadores para la formación inicial de profesores (MINEDUC, 2011).

Sin embargo, y pese a la importancia que diferentes instrumentos y organizaciones han dado a la RPM en términos de construir conocimiento y desarrollar habilidades matemáticas 
(NCTM, 1989; OCDE, 2007), no existe un consenso significativo respecto de lo que significa (XENOFONTOS; ANDREWS, 2014; ARCAVI; FRIEDLANDER, 2007; CARLSON; BLOOM, 2005; CHAPMAN, 1997; LESTER, 1994; NUNOKAWA, 2005; SCHOENFELD, 1992). En este esfuerzo, se han llegado a determinar tres grandes categorías de concepciones respecto de la RPM, a saber: como proceso, como objetivo curricular y como método de enseñanza.

Lester (1994), al realizar una revisión de la literatura respecto de la RPM desde 1970 a 1994, encuentra que, en los primeros años, el foco estuvo en describir el proceso para resolver problemas. De esta forma, Polya (1945) al observar el trabajo de los matemáticos y el suyo, observó que estos usaban diferentes heurísticas al resolver problemas, las cuales sintetizó en las siguientes fases: comprender el problema, divisar un plan, llevar a cabo el plan y mirar hacia atrás. Sin embargo, Polya no fue el primero en afirmar que la resolución de problemas implica procesos o estrategias. Dewey, a finales del siglo XIX, planteo que en cualquier proceso de creación, como es el resolver un problema, se manifiestan las siguientes etapas: identificación de la situación problemática, definición precisa del problema, análisis de los medios y fines (plan de solución), ejecución del plan, asunción de las consecuencias, evaluación de la solución, supervisión y generalización (BLANCO, 1996). Pese a que las fases de Dewey y Polya no difieren mucho, la linealidad en la presentación de las etapas ha hecho que se mal interprete su implementación (KELLY, 2006; NUNOKAWA 2005).

Teniendo esto en consideración, Mason, Burton y Stacey (1982) proponen un modelo para resolver problemas de forma circular, considerando que al resolver un problema es necesario transformar la situación de tal forma que pueda ser aplicado el conocimiento del resolutor. Este planteamiento lo reafirma Schoenfeld (1985), quien entiende que el proceso de resolver problemas supone la determinación de caminos, los que pueden ir en zig zag, con marchas hacia atrás y hacia delante. A partir de aquí, Schoenfeld plantea 4 fases para la RPM: Análisis, que implica trazar un diagrama si es posible, examinar casos particulares, simplificar el problema; Exploración, que implica examinar problemas equivalentes, examinar problemas ligeramente modificados, examinar problemas ampliamente modificados; Ejecutar, que implica llevar a cabo las estrategias que se analizaron en el proceso de Exploración; y Comprobación de la solución obtenida, que implica verificar la solución, verificar los criterios generales del problema.

En la misma revisión realizada por Lester (1994), se declara que en la década de los 80 el foco cambió hacia la identificación de los aspectos que, al resolver problemas, determinaban el éxito de la resolución, con el objetivo de lograr que otros mejoraran su 
capacidad para resolver problemas. En este sentido, Schoenfeld (1987) junto con determinar fases en el proceso de resolución, identificó conductas homogéneas relacionadas a las características del modo en que estos actuaban al abordar el problema.

De este modo, se logró llegar a la conclusión sobre la necesidad de algunos aspectos mínimos para la resolución de problemas: Conocimiento (conjunto de conocimientos matemáticos básicos y precisos para enfrentarse a los problemas), Heurísticas (conjunto de estrategias y técnicas generales para resolver problemas) y Control (meta-cognición o autoregulación). Según el autor, los resolutores efectivos de problemas planifican y hacen un seguimiento de cómo van pensando e implementando el plan de resolución. De esta forma, si no se encuentran con problemas siguen con el plan, o de lo contrario reevaluan el plan y consideran alternativas.

Para aquellos resolutores que no son eficientes, Schoenfeld (2007) plantea que, por lo general, no desarrollan la etapa de control, por lo que es necesario enseñar este tipo de conductas a los estudiantes. Además, el autor plantea que el sistema de creencias que los estudiantes tienen sobre si mismo y sobre la naturaleza de las matemáticas, dadas las experiencias de estos con la disciplina, afectan la construcción de conocimiento y lo que puedan hacer o no al enfrentarse a un problema.

Al considerar, entonces, la resolución de problemas como un método de enseñanza, Nunokawa (2005) propone cuatro focos. El primero hace referencia a enfatizar la aplicación del conocimiento que el estudiante tenga a través de la resolución de problemas, con lo cual se espera que los estudiantes refuercen sus esquemas de conocimiento matemático. El segundo aspecto a considerar es tener en cuenta nuevos métodos o ideas matemáticas que hagan sentido a la situación que se aborda en el problema. Según Vila (2001) este foco tiene como objetivo el conocimiento matemático que debe alcanzar el estudiante según el currículo escolar, siendo los problemas meros subsidiarios del contenido. El tercer enfoque es enfatizar la comprensión del problema, es decir, descomponer el problema teniendo como base el conocimiento que tiene el estudiante. Por último, el cuarto enfoque tiene relación con el énfasis del proceso en sí mismo de resolución, es decir, enseñar sobre resolución de problemas. De lo anterior se espera que los estudiantes obtengan la capacidad para tratar situaciones problemas, gestionar sus propios procesos de resolución y exponer sus modos de pensamientos.

Según Kilpatrick, Swafford y Findell, (2001), la RPM se vería como una unidad de aptitud matemática, que tendría 5 aspectos a desarrollar (entendimiento conceptual, adaptabilidad de razonamiento y disposición productiva, fluidez procedimental y competencia 
estratégica). De esta forma, el NCTM (2000) considera la RPM como un objeto de enseñanza a alcanzar, siendo parte de los cinco procesos fundamentales de la matemática junto al razonamiento, demostración, comunicación y conexión. Desde esta perspectiva, la construcción de nuevo conocimiento matemático pone a la resolución de problemas en el centro de la educación (NUNOKAWA, 2005), tal como lo propone, por ejemplo, el currículum de Singapur.

Así, si bien en la literatura se observa una variedad de interpretaciones de lo que es la resolución de problemas matemáticos, estas se pueden integrar en las categorías descritas anteriormente: proceso, método y objetivo de instrucción.

\section{Metodología}

Dado que la principal pregunta de investigación apunta a indagar respecto de las creencias de los formadores de profesores sobre la resolución de problemas, se ha seleccionado una metodología cualitativa de enfoque interpretativo, basada en los principios de la Teoría Fundamentada (STRAUSS; CORBIN, 2002), ya que esta permite comprender de forma profunda las comprensiones de las personas sobre los fenómenos en los cuales son participes. De este modo, conociendo lo que piensan sobre resolución de problemas, se podrá obtener información sobre las oportunidades que proporcionan para abordarla en la formación de los futuros profesores de educación básica.

Este estudio se realizó en el marco del proyecto FONDEF D11I1109 Elaboración, validación y aplicación de instrumentos de diagnóstico de oportunidades de aprendizaje para el logro de los estándares nacionales en la formación de profesores de Educación Básica. Este proyecto tenía por objetivo la creación de un instrumento diagnóstico de las oportunidades de aprendizaje que han tenido los profesores de educación primaria en su formación inicial, con el fin de facilitar a las instituciones formadoras la innovación de este con el fin de alcanzar su logro. Dicho instrumento pudo reportar, en base al cruce de la relación teoría-práctica y los tipos de conocimiento profesional (disciplinar y sobre la enseñanza), que los estudiantes dicen tener más oportunidades de aprendizaje de tipo disciplinar, tanto teóricas (conceptos matemáticos) como prácticas (desarrollo de habilidades matemáticas) (57\% oportunidades disciplinares con una evaluación 4 versus un $23 \%$ de enseñanza), lo que revela una formación centrada en la disciplina y no en las competencias profesionales relativas a la enseñanza (ROJAS; CHANDIA; HUENCHO, 2014). 
Los participantes del estudio fueron 8 formadores de profesores de Educación Básica que hacen clases de matemática en las universidades participantes del proyecto, y fueron seleccionados de forma intencionada, siguiendo los siguientes criterios: (1) Experiencia en la formación de profesores de educación básica. Este criterio hace referencia a la cantidad de años que llevan los formadores haciendo clases de matemática en carreras de pedagogía en Educación Básica. Se consideraron formadores con más de 5 años de experiencia. (2) Experiencia en la institución formando profesores. Este criterio hace referencia la cantidad de años que llevan los formadores haciendo clases en la institución participante en el proyecto. Se consideraron 4 años de trayectoria en la institución. (3) Experiencia a cargo de cursos de matemática o didáctica de la matemática. Este criterio hace referencia a la cantidad de años que lleva el formador dictando cursos de matemática o didáctica de la matemática a estudiantes de pedagogía en educación básica. Se consideraron 4 años de experiencia.

Para profundizar, de manera efectiva, en las oportunidades que brindan los formadores de profesores a los estudiantes de pedagogía en torno a la resolución de problemas, se utilizó una metodología de viñetas, la cual consiste en poner a los entrevistados en una situación ficticia (SCHOENBERG; RAVDAL, 2000) y a partir de ella se hacer preguntas por medio de una entrevista semi-estructurada para profundizar en el fenómeno estudiado. De esta forma, los formadores de profesores tienen la oportunidad de reflexionar en torno a una situación concreta sobre la resolución de problemas y las estrategias que consideran más efectivas para generar oportunidades de aprendizaje a los estudiantes de pedagogía en torno a este tema.

Para ello, se les envió, un día antes de la entrevista, una viñeta que les proponía reflexionar sobre la forma de abordar la resolución de problemas en la formación inicial docente. La viñeta enviada a los formadores fue la siguiente: Si tuvieras la oportunidad de abordar la resolución de problemas matemáticos en el programa de Pedagogía en Educación Básica, ¿Qué propondrías? A partir de esta viñeta, en la sesión con el formador se puso el foco en cómo trabajarían con sus estudiantes resolución de problemas, para pasar posteriormente a conversar sobre sus representaciones de RPM y las justificaciones de las actividades que les proponen a sus estudiantes.

El análisis de los datos, provenientes de las entrevistas anteriores, se realizó inicialmente a través de una codificación abierta, identificando conceptos y caracterizando el fenómeno en estudio. A partir de los códigos definidos se levantaron categorías y subcategorías que permitieron agrupar conceptos similares en su naturaleza o relacionados en su significado. Estas categorías y subcategorías permitieron establecer relaciones para generar explicaciones sobre las concepciones de los formadores respecto de la resolución de 
problemas y su estudio en la formación inicial docente. Siguiendo entonces los lineamientos de la Teoría Fundamentada (STRAUSS; CORBIN, 2002), y a partir de lo anterior, se realizaron una codificación axial, que permitió relacionar las categorías a través de sus propiedades y dimensiones, y una codificación selectiva, con el propósito de refinar e integrar la teoría alrededor de un concepto central.

\section{Análisis y resultados}

Siguiendo el interés principal de este estudio, es decir, conocer las creencias que tienen los formadores de profesores sobre la resolución de problemas, mostraremos a continuación los resultados y sus evidencias, siguiendo la estructura de la entrevista semi estructurada: creencias sobre RPM, estrategias para abordar la RPM en formación inicial docente, y fundamentos del uso de dichas estrategias.

\subsection{Creencias de los formadores sobre RPM}

El doble atributo de la resolución de problemas en la formación inicial, como medio para aprender matemáticas y para aprender a enseñar matemáticas, fue mencionado por la mayoría de los formadores. Por ejemplo, uno de los formadores señala que "la resolución de problemas que en este caso que te señalo uno podría decir -Ya para que aprenda matemática, para que aprenda a enseñar matemáticas y aprenda enseñarla bien" (Entrevista 1). Este mismo formador, específicamente, indica que la resolución de problemas sirve para aprender a enseñar matemáticas: "la resolución de problema para mí también puede significar el hecho de cómo los estudiantes aprenden al enseñar esta disciplina” (Entrevista 1).

En este sentido, los formadores entrevistados sostienen que la RPM es un eje transversal en el estudio de la matemática y como medio lo consideran aplicable en cualquier momento de la clase: "la resolución de problemas primero es transversal...no solamente al inicio de la clase, si no que en el desarrollo de la clase misma” (Entrevista 2). Los formadores entrevistados reconocen la RPM como un medio para enseñar matemáticas, para aprender conceptos y contenidos; es un medio que facilita la reflexión, el análisis y la argumentación, y un medio para evaluar aprendizajes: "Mira con este problema yo quiero que los estudiantes se den cuenta, comprendan profundamente en concepto fracción" (Entrevista 1); "resolver las problemáticas que tú le vas presentando en términos del contenido que les estas enseñando...que el contenido matemático sea presentado a través de un problema” (Entrevista 
2). Específicamente, para los formadores entrevistados, la resolución de problemas es también un medio de evaluación: "a ti te permite tener evidencia de que si el niño comprende, si aplica, si es capaz de establecer una conjetura y verificarla, establecer a mi juicio...yo quiero saber si el niño sabe dividir decimales y te ocupa la resolución de problemas para ello" (Entrevista 1).

\subsection{Estrategias para abordar la RPM}

Para lograr que los estudiantes de pedagogía vivencien procesos de resolución de problemas, los formadores, por una parte, modelan en sus clases de matemáticas actividades relacionadas con el aula escolar, y, por otra, resuelven problemas en que les piden a sus estudiantes que utilicen diversas estrategias para resolver los problemas, generando procesos de discusión con el propósito de contrastar dichas estrategias en torno a su eficacia. En el primero de los casos, cuando los formadores entrevistados relatan cómo enseñan a enseñar a resolver problemas, se refieren a estrategias basadas en modelar dicha enseñanza. Indican que - entre las estrategias que utilizan para modelar su enseñanza - presentan a los estudiantes la tarea de diseñar o plantear problemas y adaptarlos situaciones-problemas para diferentes niveles de enseñanza: "Bueno al principio lo hago con ellos pero tendrían que ver cómo lo adaptarían a quinto básico" (Entrevista 4).

Otro tipo de actividad, que se mencionó con frecuencia, es el diseño y preparación de actividades o secuencias de actividades para la resolución de problemas matemáticos. Al efectuar el estudio de una noción matemática, los formadores de profesores solicitan a los estudiantes que elaboren problemas para una actividad o secuencia de actividades que podría ser trabajada en el aula escolar. Este proceso, según los formadores, posibilita también el análisis del currículum. Por ejemplo, señalan:

[...] tú vas a enseñar la resolución de problemas o vas a implementar con la resolución de problemas la enseñanza de tal contenido. ¿Cómo lo harías? ¿Qué pasos seguirías? Tales... Ya y con tus estudiantes, ¿qué pasos seguirías? Tales... Ya y ahora, tus estudiantes siguiendo esos pasos, ¿qué van a hacer? ¿Qué habilidades van a desarrollar? (Entrevista 4).

En la misma línea de preparación de la enseñanza, los formadores describen estrategias en las que piden a sus estudiantes que diseñen clases donde se utilicen problemas contextualizados, en que se use tanto la contingencia como las características del medio en que se realizará la clase:

Te das cuenta, es que igual ahí hay un problema contextualizándote que podrían usar, entonces ellos podrían plantear un problema, ya, resolvamos, cuánto nos 
costaría pintar nuestras casas. En cuanto a pinturas, costos, pero una casa que tiene tales dimensiones. Porque ahí ya lo llevas al contexto y evitas el que te diga para qué (Entrevista 4).

En el segundo de los casos, cuando modelan maneras de resolver problemas, les piden a sus estudiantes que utilicen diferentes estrategias de solución y que construyan las soluciones de manera colectiva: "actividades que permitan que el estudiante, buscando distintas estrategias, pueda resolver las problemáticas" (Entrevista 2). En este sentido, para modelar la resolución de problemas, los formadores entrevistados ponen cuidado en elegir distintos tipos de problemas en función de la noción matemática que se desea trabajar: "actividades que permitan que el estudiante, buscando distintas estrategias, pueda resolver las problemáticas...las estrategias de solución van en función de lo que se espera adquirir" (Entrevista 2). Así, en algunos casos seleccionan problemas para aplicar conceptos, en otros buscan problemas que inviten a descubrir diferentes caminos de solución, e incluso problemas que desafíen, que requieran búsqueda de información adicional, pero que siempre su solución esté al alcance de los estudiantes.

Los formadores reconocen utilizar diferentes tipos de problemas: abiertos, problemas matemáticos, problemas sin enunciado, sin preguntas, sin datos: "son problemas de la vida...problemas sin enunciado, problemas sin pregunta, que el estudiante complete con la pregunta, problemas sin datos...o una frase numérica a partir de frase numérica que cree un contexto" (Entrevista 2). Sin embargo, para los formadores, las oportunidades de aprendizaje en resolución de problemas dependen de si los enunciados de los problemas son desafiantes y corresponden a problemas no rutinarios. Por ejemplo, señalan: "un enunciado tiene que ser un desafío, no una simple lectura donde se extraigan datos, pero tampoco desafío que no se pueda hacer, que no implique que se necesita conocimientos que se ponen en juego, pero para eso tú tienes que enseñarles a modelar primero" (Entrevista 6). En el mismo sentido, de modelar la resolución de problemas, los formadores entrevistados se apoyan en el uso de preguntas guía que utilizan para conversar con sus estudiantes durante los procesos de resolución: cómo se hace, qué significa, cómo lo enfrentan, por qué creen que está bien lo que hicieron: "Tienes que decirles ¿qué ven ustedes, cómo lo enfrentarían? ¿Por qué creen que está bien? ¿Por qué creen que está mal?” (Entrevista 3). Respecto de cómo resolver problemas, los formadores entrevistados aluden a diversos modelos o marcos teóricos referidos a las etapas en la resolución de problemas, indicando que la resolución de problemas se trabaja en etapas, fases o pasos, que pueden ser modificados o adaptados por quién lo 
realiza: "después de eso empezamos a trabajar con distintos modelos, yo les presento distintos modelos. Ellos se apropian de uno" (Entrevista 4).

Al trabajar la resolución de problemas en la formación inicial docente, los formadores declaran que, en sus universidades, utilizan la resolución de problemas como medio para el logro de otros aprendizajes, alineando sus programas con las con el currículum escolar:

[...] vas a las bases curriculares tú le empiezas a mostrar qué diferencias hay entre un problema...el estudiante tiene que ser capaz de tener claro cuál es la demanda curricular ¿cuáles son los problemas que responden a ese objetivo de aprendizaje declarado en el programa pero también con la habilidad que dicen las bases curriculares, por ejemplo qué dice que el estudiante tiene que argumentar en ese $2^{\circ}$ básico? (Entrevista 1).

Así, los formadores declaran que preparan a sus estudiantes para que lo trabajen, a su vez, con sus alumnos. Luego, los estudiantes de pedagogía deberían apropiarse o desarrollar un modelo para enfrentar la RPM, de modo que sean capaces de resolver los problemas con diversas metodologías, con diferentes algoritmos:

\begin{abstract}
Entonces al final tú les vas enseñando una estrategia, va a llegar un momento en que el niño va a llegar solo y que ya a lo mejor no van a estar los ocho pasos, pero cuando tenga dudas va a decir, hay que primero hacer esto, luego esto y luego esto otro...Entonces, yo le decía ya hagamos el modelo. Entonces ella me decía, profesora ¿qué pasa si ellos hacen su propio modelo? Esa es la idea (Entrevista 4).
\end{abstract}

En este sentido, los formadores afirman que usan la resolución de problemas como medio, debido a que ella sirve para desarrollar conceptos, integrarlos, aplicarlos en situaciones concretas. Al contextualizar su aplicación ayudan a dar sentido a entidades abstractas, dando sentido a las matemáticas como herramienta de interpretación de la realidad: "Entonces cuando trabajas esos elementos que son como tan importantes que él [estudiante] tenga súper claro lo que está trabajando, el concepto matemático, y lo presentas a través de una situación problemática" (Entrevista 2).

En concordancia con ello, los formadores afirman que la resolución de problemas permite acceder a los errores típicos de los estudiantes, a develarlos, y, por tanto, permite presentar estrategias para abordar esos errores: "es bueno que se encuentren con las dificultades típicas, y que si ellos no las tienen vean que otros si las pueden tener...si es que ellos no se han visto enfrentados a ese error probablemente no lo hacen consiente y después no le logran ver el error" (Entrevista 3).

Finalmente, un formador señala que la resolución de problemas, curricularmente - en referencia al programa de formación inicial de su universidad - está en terreno de nadie: "Y en ese sentido la resolución de problemas está como en terreno de nadie. Ha sido una de las críticas que yo he hecho al tema, o sea, quien finalmente asume el trabajo como resolución de 
problemas" (Entrevista 2). De hecho, uno de los formadores entrevistados indica que trabaja la resolución de problemas como un tópico especial, en un tiempo acotado y específico del semestre y no como una estrategia transversal del programa: "lo que hago es ver sobre el poco tiempo que tenemos, es decir 12 o 16 semanas. Ahí de esas 16 semanas le dedicas 3 semanas a este tópico" (Entrevista 1).

\subsection{Fundamentos del uso de estrategias para abordar la RPM}

En la fundamentación que hacen los formadores sobre el uso de determinadas estrategias para abordar la RPM, aparece la habilidad de razonamiento matemático como un componente fuertemente relacionado a la resolución de problemas, dándoles una alta valoración, en contraste con el uso de fórmulas y procedimientos estandarizados. Señalan que el proceso que se lleva a cabo al resolver un problema implica que los estudiantes razonen en torno a los conocimientos que poseen de la disciplina, lo que es muy positivo para su formación. Por ejemplo, indican: "pero siempre y cuando sepan entender esa fórmula y pensar matemáticamente y en el fondo tengan una base de razonamiento que les permita aprender fácilmente otros contenidos" (Entrevista 7).

En este sentido, los formadores entrevistados declaran que el conocimiento matemático se desarrolla a través de la resolución de problemas, específicamente a través de la búsqueda de diferentes estrategias:

[...] el desarrollo del conocimiento matemático se va a desarrollar si o si hay una buena estrategia problemática, no solamente al inicio de la clase, si no que en el desarrollo de la clase misma a través de actividades que permitan que el estudiante, buscando distintas estrategias, pueda resolver las problemáticas que tú le vas presentando en términos del contenido que les estas enseñando (Entrevista 2).

En el sentido de la RPM como medio, los formadores señalan que esta sirve para desarrollar diferentes habilidades: "entendemos que el desarrollo del conocimiento matemático se va a desarrollar si o si hay una buena estrategia problemática...la matemática es una herramienta para resolver esos tipos de problemas" (Entrevista 2).

Por otra parte, los formadores fundamentan el carácter longitudinal de la resolución de problemas y su relación con la secuencia curricular en que se implementa:

[...] no puedes pretender que en un tercero, cuarto básico que el niño por generaciones espontánea te resuelva problemas así como -Oye mira yo les vengo a proponer esto-, pero ¿qué paso con ese chiquitito en segundo?, qué paso con ese chiquitito en primero A? y ¿qué paso en kínder y en pre-kínder con ese niño?...el estudiante tiene que ser capaz de tener claro cuál es la demanda curricular" (Entrevista 1). 
En este sentido, para uno de los formadores entrevistados, el solo hecho de resolver problemas no asegura una preparación del futuro docente:

[...] para qué van a estar preparados, para hacerlo transversalmente. ¿Cómo? Solo si son conscientes de lo que ocurrió con ellos, ¿te fijas? Si solo los sometiste a la experiencia de resolver problemas transversalmente con estrategias, con teoría, con todo lo que tú quieras, no necesariamente están preparados para enseñarlo (Entrevista 7).

Así, enseñar a enseñar a resolver problemas en la formación inicial docente, les permite -a los formadores entrevistados - utilizar el error como una oportunidad de aprendizaje: "es bueno que se encuentren con las dificultades típicas, y que si ellos no las tienen vean que otros si las pueden tener...si es que ellos no se han visto enfrentados a ese error probablemente no lo hacen consiente y después no le logran ver el error" (Entrevista 3).

En definitiva, los formadores entrevistados fundamentan en parte sus decisiones respecto de cómo enseñar resolución de problemas a estudiantes de pedagogía, en referencia a que los estudiantes aprenden matemáticas haciendo matemáticas, aprenden a resolver problemas, resolviendo problemas: "Yo creo que la capacidad de análisis no se las puedes enseñar diciéndoles lo que es el análisis, tienen que darse cuenta ellos como partir analizando, se desarrolla en la práctica... que un chiquillo tenga que indagar para poder plantear el problema y poder resolverlo" ( Entrevista 2).

\section{Discusión y conclusiones}

Al analizar las respuestas de los formadores de profesores se observa que la concepción sobre resolución de problemas no es única entre ellos, al igual que en la literatura (ANDREWS; XENOFONOS, 2009, 2014; CARLSON; BLOOM, 2005; CHAPMAN, 1997; LESTER, 1994). Entre las concepciones de los formadores coexisten, al menos, dos definiciones fuertes en sus declaraciones, a saber: RPM como método de enseñanza y RPM como objetivo curricular. En menor medida, se observa en las declaraciones de los formadores la definición de RPM como un proceso (XENOFONOS; ANDREWS, 2014 ; LESTER, 1994). A continuación se exponen las relaciones encontradas.

Los formadores de profesores principalmente señalan a la resolución de problemas como medio para aprender matemáticas, como conceptos y contenidos y para aprender a enseñar matemáticas. Vila (2001) y Nunokawa (2005) declaran que este enfoque para enseñar matemática considera a la RPM como mero subsidiario del conocimiento o habilidades que el profesor quiere que los estudiantes alcancen. Sin embargo, también reconocen que la RPM 
facilita la reflexión, el análisis y la argumentación. Schoenfeld (1987, 2012), al observar a los resolutores de problemas, destaca la reflexión como una de las características que hace la diferencia entre los que resuelven problemas de forma eficiente de los que no. La capacidad de reflexionar y analizar el plan y las estrategias, evaluando el éxito de estas, y las posibles reconsideraciones, son habilidades que él subraya como deseables de ser enseñadas a los estudiantes para mejorar su capacidad para resolver problemas. Para ello, los formadores de profesores declaran usar estrategias de modelación de actividades de aula escolar, solicitando a sus estudiantes que preparen actividades de enseñanza, diseñen tareas, planteen problemas, los adapten y les den un contexto cercano a la vida de los alumnos, identificando errores típicos al abordar los problemas, para luego diseñar formas de abordarlos. Lo anterior, exige a los estudiantes de pedagogía reflexionar y analizar las diferentes formas de abordar un problema, pero desde las perspectiva de los niños y niñas. De esta forma, la RPM, para estos formadores, es medio y fin para desarrollar diferentes habilidades matemáticas y de enseñanza.

Un aspecto interesante que surge en el análisis, es que además de considerar la RPM como medio, también consideran que tiene una función de mecanismo de evaluación de aprendizajes, pudiendo ser usado en cualquier momento de una clase. Nunokawa (2005) declara que esta acepción enfatiza la aplicación del conocimiento que el estudiante tenga a través de la resolución de problemas, siendo a la vez medio y causa, dado que los estudiantes deberán gestionar sus propios procesos de resolución y exponer sus modos de pensamientos.

Los formadores de profesores declaran, también, que entienden que deben enseñar a enseñar a resolver problemas a los estudiantes de pedagogía. Esto coincide con el enfoque de la resolución de problemas como un objetivo curricular, tal como lo plantea Kilpatrick, Swafford y Findell, (2001), Vila (2001), Nunokawa (2005), y Schoenfeld (2012). En este sentido, los formadores de profesores declaran que presentan a sus estudiantes de pedagogía diferentes tipos de problemas, con enunciados desafiantes, en que haya que usar diferentes estrategias de solución. Los estudiantes los resuelven individualmente y en grupos, orientados por el formador mediante preguntas guías, invitando a usar estrategias y modelos de solución. Sin embargo, y aunque sea un objetivo particular a lograr, los formadores reconocen que la resolución de problemas es una habilidad transversal, que se desarrolla en el tiempo.

Finalmente, y aunque los formadores declaran que la resolución implica un proceso que está compuesto por etapas o fases, tan solo se refieren a la necesidad de utilizar diversas estrategias. Si bien, esto es una parte fundamental en la resolución de problemas 
(SCHOENFELD, 1987), el análisis, la ejecución y la misma comprobación de la estrategia e interacción de estas fases no se observan en las declaraciones de los formadores de profesores.

\section{Agradecimientos}

Este proyecto ha sido realizado en el marco del proyecto FONDEF D11I1109 Elaboración, validación y aplicación de instrumentos de diagnóstico de oportunidades de aprendizaje para el logro de los estándares nacionales en la formación de profesores de Educación Básica - financiado por la Comisión Nacional de Investigación Científica y Tecnológica CONICYT y desarrollado en la Pontificia Universidad Católica de Chile.

\section{Referencias}

ARCAVI, A.; FRIEDLANDER, A. Curriculum developers and problem solving: the case of Israeli elementary school projects. ZDM: The International Journal on Mathematics Education, Monterrey, v. 39, n. 5, p. 355-364, 2007.

BALL, D. L.; THAMES, M. H.; PHELPS, G. Content Knowledge for Teaching. What Makes it Special? Journal of Teacher Education, New York, v. 59, n. 5, p. 389-407, 2008.

BLANCO, J. L. . La resolución de problemas. Una revisión teórica. Suma, Barcelona, v. 21, p. 11-20, 1996.

BOYD, D.; GROSSMAN, P.; LANKFORD, H.; LOEB, S.; WYCKOFF, J. Teacher Preparation and Student Achievment. Educational Evaluation and Policy Analysis, Washington, v. 31, p. 416-440, 2009.

CAMACHO, M.; SOCAS, M. M.; HERNÁNDEZ, J. An analysis future mathematics teachers' conceptions and attitude towards mathematics. International Journal of Mathematics Education in Science and Technology, Londres, v. 24, n. 3, p. 317-324, 1998.

CARLSON, M. P.; BLOOM, I. The cyclic nature of problem solving: An emergent multidimensional problem-solving framework. Educational Studies in Mathematics, Ámsterdam , v. 58, n. 1, p. 45-75, 2005.

CHAPMAN, O. Mathematics teacher educator's learning from research on their instructional practices. In: B. JAWORSKI; T. WOOD (Ed.). The Mathematics Teacher Educator as a Developing Professional. Handbook of Mathematics Teacher Education. Rotterdam: Sense Publishers, 2008. p. 115-134.

CHAPMAN, O. Metaphors in the teaching of mathematical problem solving. Educational Studies in Mathematics, Ámsterdam, v. 32, n. 3, p. 201-228, 1997.

CHRISTIANSEN, B.; WALTER, G. Task and activity. In: CHRISTIANSEN, B.;; HOWSON, A. G.; OTTE, M. (Ed.). Perspectives on Mathematics Education. Londres, Reidel: Reidel Publishing, 1986. p. 243-307. 
CISTERNAS, T. La investigación sobre formación docente en Chile. Territorios explorados e inexplorados. Calidad en la Educación, Santiago de Chile, v. 35, p. 131-164, 2011.

COCHRAN-SMITH, M.; FRIES, K.; ZEICHNER, K. Estudio sobre la formación del profesorado en los Estados Unidos: descripción del informe del comité de la American Educational Research Association (AERA) sobre Calidad de la Educación. Investigación y formación del profesorado. Revista de Educación, Washington, v. 35, n. 340, p. 87-116, 2006.

DEULOFEU, J.; FIGUEIRAS, L.; PUJOL, R. De lo provisible a lo inesperado en un contexto de resolución de problemas. Uno. Revista de Didáctica de la Matemática, Barcelona, v. 58, p. 84-97, 2011.

DEULOFEU, J.; MÁRQUEZ, C.; SANTMARTÍ, N. Formar profesores de secundaria: la experiencia de la Universitat Autònoma de Barcelona. Cuadernos de Pedagogía, Madrid,v. 404, p. 80-84, 2010.

DOMÍNGUEZ, M.; MECKES, L. Análisis y propuestas para la acreditación de pedagogías en Chile. Calidad en la Educación, Santiago de Chile, v. 34, p. 165-183, 2011.

FELMER, P.; PERDOMO, J. La Resolución de Problemas en la Matemática Escolar y en la Formación Inicial Docente. Informe Proyecto FONIDE N $^{\circ}$ 721209. Santiago: Ministerio de Educación, 2014.

GELLERT, U. La formación docente entre lo teórico y lo práctico. In: . GÓMEZ-CHACÓN, I. M.; PLANCHART, E. (Coord.). Educación Matemática y Formación de Profesores: Propuestas para Europa y Latinoamérica. Bilbao: Universidad de Deusto, 2005. p. 73-83.

GINÉ, C.; DEULOFEU, J. Conocimientos y Creencias en torno a la Resolución de Problemas de Profesores y Estudiantes de Profesor de Matemáticas. Bolema, Rio Claro, v. 28, n. 48, p. 191-208, 2014.

GÓMEZ-CHACÓN, I. M.. Tendencias y retos en formación de profesores en Matemáticas. Vivir el presente y crear futuro en la cooperación Europa-Latinoamérica. In: GÓMEZ-CHACÓN I. M.; PLANCHART, E. (Coord.). Educación Matemática y Formación de Profesores: Propuestas para Europa y Latinoamérica. Bilbao: Universidad de Deusto, 2005. p. 15-31.

JAWORSKI, B. Mathematics teacher educator learning and development. In: JAWORSK, B. I.; WOOD, T. (Ed.). The Mathematics Teacher Educator as a Developing Professional. Handbook of Mathematics Teacher Education. Rotterdam: Sense Publishers, 2008. p. 1-13.

KELLY, C. A. Using manipulatives in mathematical problem solving: A performance-based analysis. The Mathematics Enthusiast, Montana, v. 3, n. 2, p. 184-193, 2006.

KILPATRICK, J.; SWAFFORD, J.; FINDELL, B. Adding it up: Helping children learn mathematics. Washington: National Academy Press, 2001.

LESTER, F. K. Musings about mathematical problem-solving research: 1970-1994. Journal for Research in Mathematics Education, Reston, v. 25, n. 6, p. 660-67, 1994.

LILJEDAHL, P.; OESTERLE, S.; BERNÈCHE, C. Stability of beliefs in mathematics education: a critical analysis. Nordic Studies in Mathematics Education, Gothenburg,v. 17, n. 3-4, p. 2340,2012 .

MASON, J.; BURTON, L.; STACEY, K. Thinking mathematically. London: Addison-Wesley, 1982. 
MINEDUC. Bases Curriculares para la Educación Básica. Santiago: Ministerio de Educación, 2012.

MINEDUC. Estándares Orientadores para Egresados de Carreras de Pedagogía en Educación Básica. Santiago: Ministerio de Educación, 2011.

MINEDUC. Informe de la Comisión sobre Formación Inicial Docente (documento no oficial). Santiago: Ministerio de Educación, 2005.

NCTM. Curriculum and evaluation standards for school mathematics. Reston: National Council of Teachers of Mathematics, 1989.

NCTM. Principios y Estándares para la Educación Matemática. Sevilla: Thales, 2000.

NUNOKAWA, K. Mathematical problem solving and learning mathematics: What we expect students to obtain. The Journal of Mathematical Behaviour, New Jersey, v. 24, n. 3-4, p. 325-340. 2005.

OCDE; BANCO MUNDIAL. Revisión de Políticas Nacionales de Educación: La educación Superior en Chile. Santiago: OCDE, 2009.

OCDE. Science competencies for tomorrow's world. Paris: Organisation for Economic Cooperation and Development, 2007.

OP’T EYNDE, P.; DE CORTE, E.; VERSCHAFFEL, L. Framing students'mathematics-related beliefs: a quest for conceptual clarity and a comprehensive categorization. In: LEDER, G.; PEHKONEN, E.; TÖRNER, G. (Ed.). Beliefs: a hidden variable in mathematics education? Dordrecht: Kluwer, 2002. p. 13-37.

POLYA, G. How to solve it: A new aspect of mathematics. New Jersey: Princeton University Press, 1945.

PUTNAM, R.; BORKO, H. What do new views of knowledge and thinking have to say about research on teacher learning? Educational Researcher, Washington, v. 29, p. 4-15, 2000.

RANDI, J.; CORNO, L. Teacher innovations in self-regulated learning. In: PINTRICH, P.; BOEKAERTS, M.; ZEIDNER, M. (Ed.). Handbook of self-regulation. Orlando: Academic, 2000. p. 651-685.

ROJAS, F.; CHANDIA, E.; HUENCHO, A. Learning opportunities in primary mathematics teacher education. In: OESTERLE, S.; NICOL, C.; LILJEDAHL, P.; ALLAN, D. (Ed.). Proceedings of the Joint Meeting of PME 38 and PME-NA 36, Vancouver, v. 6, p. 211, 2014.

ROJAS, F.; DEULOFEU, J. El formador de profesores de matemática: un análisis de las percepciones de sus prácticas instruccionales desde la «tensión» estudiante-formador. Enseñanza de las Ciencias, Barcelona, v. 33, n. 1, p. 47-61, 2015.

SCHOENBERG, N. E.; RAVDAL, H. Using vignettes in awareness and attitudinal research. International Journal of Social Research Methodology, Londres, v. 3, n. 1, p. 63-74, 2000.

SCHOENFELD, A. H. Assessing mathematical proficiency. New York: Cambridge University Press, 2007.

SCHOENFELD, A. H. Learning to think mathematically: Problem solving, metacognition, and sense making in mathematics. In:GROUWS, D. (Ed.). Handbook of research on mathematics teaching and learning. New York: MacMillan, 1992. p. 334-370. 
SCHOENFELD, A. Mathematical Problem Solving. New York: Academic Press, 1985.

SCHOENFELD, A. H. Cognitive science and mathematics education: an overview. In:

SCHOENFELD, A. H. (Ed.). Cognitive Science and Mathematics Education. London: Lawrence Erlbaum Associates, 1987. p. 1-31.

SOTOMAYOR, C.; GYSLING, J. Estándares y Regulación de Calidad de la Formación de Profesores: Discusión del Caso Chileno desde una Perspectiva Comparada. Calidad en la Educación, Santiago, v. 35, p. 91-129, 2011.

STRAUSS, A.; CORBIN, J. Bases de la investigación cualitativa. In: STRAUSS, A.; CORBIN, J. Técnicas y procedimientos para desarrollar la teoría fundamentada. Bogotá: Universidad de Antioquia, 2002.

TATTO, M. T.; PECK, R.; SCHWILLE, J.; BANKOV, K.; SENK, S. L.; RODRIGUEZ, M.; INGVARSON, L.; RECKASE, M.; ROWLEY, G. Policy, Practice, and Readiness to Teach Primary and Secondary Mathematics in 17 Countries. Findings from the IEA Teacher Education and Development Study in Mathematics (TEDS-M). Amsterdam: International Association for the Evaluation of Educational Achievement (IEA), 2012.

VILA, A. Resolució de problemes de matemàtiques: Identificació, origen i formació dels sistemes de creences en l'alumnat. Alguns efectes sobre l'abordatge dels problemes. 2001. 659 f. Tesis Doctoral (Doctorado en didáctica de las matemáticas) - Facultad de Educación, Universitat Autònoma de Barcelona, Bellaterra, 2001.

WATSON, A.; MASON, J. Taken-as-shared: a review of common assumptions about mathematical tasks in teacher education. Journal of Mathematics Teacher Education, Londres, v. 10, p. 205-215, 2007.

WILSON, M. Y COONEY, T. Mathematics teacher change and developments. The role of beliefs. In: LEDER, G.; PEHKONEN, E.; TÖRNER, G. (Ed.). Beliefs: a hidden variable in mathematics education? Dordrecht: Kluwer, 2002. p. 127-147.

XENOFONTOS, C.; ANDREWS, P. Defining mathematical problems and problem solving: prospective primary teachers' beliefs in Cyprus and England. Mathematics Education Research Journal, Amsterdam,v. 26, n. 2, p. 279-299, 2014.

YEE, L. P. Mathematics for Teaching or Mathematics for Teachers? The Mathematics Educator, Singapur v. 16, n. 2, p. 2-3, 2006.

ZAPATA, M. A.; BLANCO, L. J.; CAMACHO, M. Análisis de las concepciones de los estudiantes para professor sobre las matemáticas y su enseñanza-aprendizaje. Bolema, Rio Claro, v. 26, n. 44, p. 1443-1466, 2012.

ZASLAVSKY, O. Mathematics-related task, teacher education, and teacher educator. Journal of Mathematics Teacher Education, Londres, v. 10, n. 4-6, p. 433-440, 2007.

Submetido em Junho de 2015. Aprovado em Outubro de 2015. 